\title{
Should the frequency, severity, or both response scales be used for multi-item dental patient-reported outcome measures?
}

\author{
Swaha Pattanaik ${ }^{\text {Corresp., } 1}{ }^{,}$Mike T. John ${ }^{1}$, Seungwon Chung ${ }^{2}$, San Keller ${ }^{3}$ \\ ${ }^{1}$ Department of Diagnostic and Biological Sciences, School of Dentistry, University of Minnesota, Minneapolis, Minnesota, United States \\ 2 Department of Educational Psychology, College of Human Development, University of Minnesota, Minneapolis, Minnesota, United States \\ 3 American Institutes of Research, Chapel Hill, North Carolina, United States \\ Corresponding Author: Swaha Pattanaik \\ Email address: swahapattanaik@gmail.com
}

Background: The Oral Impacts on Daily Performances (OIDP) index asks the respondents to indicate both, the frequency and severity of the impact. However, it is not clear if the two scaling methods are correlated, and if using one scale is sufficient. The purpose of the study was to investigate the correlation between frequency and severity rating scales of the OIDP instrument, and whether only one of the rating scales can be used instead of both.

Methods: A battery of patient-reported outcome questionnaires were administered to a consecutive sample of adult dental patients from HealthPartners dental clinics in Minnesota $(\mathrm{N}=2,115)$. Only those who responded to any of the OIDP items were included in the analysis for this study ( $N=873$ ). We assessed correlations between the frequency and severity scales for all OIDP items, and for the summary scores of the two OIDP response scales. We additionally fit a categorical structural equation model (SEM) (or an item factor analysis model) and examined the correlation between two latent variables (Frequency and Severity).

Results: The correlation estimates for all OIDP items were greater than 0.50 , indicating large correlations between the frequency and severity scores for each OIDP item. The correlation estimate between the two summary scores was 0.85 (95\% Cl: $0.82-0.86)$. When we calculated the correlation coefficient using a latent variable model, the value increased to 0.96 ( $95 \% \mathrm{Cl}: 0.93-1.00)$.

Conclusion: Our study findings show that OIDP frequency and severity scores are highly correlated, which indicates the use of one scale only. Based on previous evidence, we recommend applying the frequency rating scale only in research and clinical settings. 
1 Should the frequency, severity, or both response scales be used for multi-item dental

2 patient-reported outcome measures?

3

4 Swaha Pattanaik ${ }^{1}$, Mike T. John ${ }^{1 *}$, Seungwon Chung ${ }^{2}$, San Keller ${ }^{3}$

5

6

$7{ }^{1}$ Department of Diagnostic and Biological Sciences, School of Dentistry, University of

8 Minnesota, Minneapolis, Minnesota

9

$10{ }^{2}$ Department of Educational Psychology, College of Human Development, University of

11 Minnesota, Minneapolis, Minnesota

12

$13{ }^{3}$ American Institutes of Research, Chapel Hill, North Carolina

14

15 Correspondence

16 Swaha Pattanaik, Department of Diagnostic and Biological Sciences,

17 University of Minnesota, School of Dentistry,

18515 Delaware Street Southeast,

19 Minneapolis, MN 55455-0348.

20 Email: swahapattanaik@gmail.com

21

22 


\section{Abstract}

25 Background: The Oral Impacts on Daily Performances (OIDP) index asks the respondents to 26 indicate both, the frequency and severity of the impact. However, it is not clear if the two scaling 27 methods are correlated, and if using one scale is sufficient. The purpose of the study was to 28 investigate the correlation between frequency and severity rating scales of the OIDP instrument,

29 and whether only one of the rating scales can be used instead of both.

30 Methods: A battery of patient-reported outcome questionnaires were administered to a consecutive sample of adult dental patients from HealthPartners dental clinics in Minnesota $(\mathrm{N}=2,115)$. Only those who responded to any of OIDP items were included in the analysis of this study $(\mathrm{N}=873)$. We assessed correlations between the frequency and severity scales for all OIDP items, and for the summary scores of the two OIDP response scales. We additionally fit a categorical structural equation model (SEM) (or an item factor analysis model) and examined the correlation between two latent variables (Frequency and Severity).

Results: The correlation estimates for all OIDP items were greater than 0.50 , indicating large correlations between the frequency and severity scores for each OIDP item. The correlation estimate between the two summary scores was 0.85 (95\% CI: 0.82-0.86). When we calculated the correlation coefficient using a latent variable model, the value increased to 0.96 (95\% CI:

$410.93-1.00)$.

42 Conclusion: Our study findings show that OIDP frequency and severity scores are highly correlated, which indicates the use of one scale only. Based on previous evidence, we recommend applying the frequency rating scale only in research and clinical settings. 


\section{Background}

A dental patient-reported outcome measure (dPROM) is an instrument, questionnaire, scale, or survey that captures patient-reported outcomes (dPROs) for adult dental patients and expresses it numerically in a score [John, 2018; Paulson et al., 2021]. The numerical score captures the oral diseases' impact [John, 2018; Paulson et al., 2021]. Oral health-related quality of life (OHRQoL) is arguably the most important $\mathrm{dPRO}$ as it measures the impact of oral diseases on people and their well-being and life quality [Paulson et al., 2021; Mittal et al., 2019]. Consequently, several dPROMs are available to assess OHRQoL and they use various response scales to characterize the impact [Mittal et al., 2019]. Frequency and severity are the two most common ways in which dPROMs assess the quality of the impact [Paulson et al, 2021; Reissmann, 2021]. While dPROMs such as the Oral Health Impact Profile (OHIP) assess the temporal quality of an impact such as frequency, that is, how often the impact occurred [Slade \& Spencer, 1994], other dPROMs such as the Orofacial Esthetic Scale (OES) assess intensity or severity of the impact [Larsson et al., 2010]. Overall, researchers seem to agree that an ordinal response scale, i.e., several steps on a frequency or severity spectrum, is preferred to a dichotomous response scale, e.g., yes-no: an ordinal response provides more variability in the score distribution and hence leads to greater score precision. However, which feature of the impact, whether frequency or severity, is better, is not clear.

Theoretically, assessing both frequency and severity would be more comprehensive than assessing either alone; yet, assessing both increases administrative and patient burden. The patients have to recall not only the frequency of the impact but also its severity. This requires more cognitive processing. For instance, the patients need to decide what severity to report, the severity they are experiencing in the moment, average intensity over a certain period of time, or the highest intensity in this period [Reissmann, 2021]. Research shows that respondents find remembering details of events from the previous day difficult, and thus averaging intensity over even a day may be quite cognitively demanding [Organisation for Economic Co-operation and Development, 2013]. Reissmann recommends using one response format for each item and not changing response formats between items [Reissmann, 2021].

Given the burden and complications involved in including two response scales for each impact, we sought to determine the degree to which the two response scales provided redundant information. If the information they provide is redundant, it would be possible to use just one 
85 response scale and so to ameliorate the problems associated with dual response scales. The Oral 86 Impacts on Daily Performances (OIDP) index, a widely used socio-dental indicator, is unique in

87 that it quantifies oral impacts by asking respondents to indicate both the frequency and severity 88 of the impacts [Adulyanon \& Sheiham, 1997]. To answer the question of whether impact 89 frequency or impact severity are redundant, existing OIDP data offer a natural, cost-effective 90 opportunity. If scores based on frequency responses would not be highly correlated with those based on severity responses, we would assume that both response scales are required because they measure different things. If frequency and severity were highly correlated, they measure the same thing, and only one quality is necessary in future studies.

Results of such a study comparing frequency and severity of oral health impacts would have both narrow and broad implications. From a narrow point of view, using a single response scale (that is, either frequency or severity but not both) for the OIDP would help reduce costs and time to administer the instrument. From a broader point of view, these findings would advance our understanding of how to design the response format for dPROMs in general. For example, findings for the OIDP can likely be generalized to other OHRQoL instruments, as OIDP is highly correlated with other OHRQoL instruments [Robinson et al., 2003; John et al., 2016]. Despite these remarkable practical implications, we are aware of no other studies that have investigated if the OIDP, or any other dPROMs, require both frequency and severity response scales. The purpose of the study was to investigate the correlation between frequency and severity rating scales of the OIDP instrument, and whether only one of the rating scales can be used instead of both.

Methods

\section{Participants, study design and setting}

This is a secondary research study using data from another cross-sectional study

109 (Simancas-Pallares et al., 2018, 2020). The researchers in the original study used a consecutive 110 sampling strategy to recruit a total of 2,115 English - and Spanish- speaking adult dental patients

111 from HealthPartners dental clinics in Minnesota, USA, between July 2014, and April 2016. A

112 battery of dPROMs consisting of items from the OIDP, Oral Health Impact Profile (OHIP), and

113 General Oral Health Assessment Index (GOHAI), were administered to the participants. They

114 filled out the self-administered questionnaires at home and sent them back to the researchers. All 
115 participants received $\$ 50$ incentives for their participation. Please refer to the previous research

116 studies (Simancas-Pallares et al., 2018, 2020) for further details about data collection and

117 recruitment. This research was conducted in accordance with accepted ethical standards for

118 human-patient research practice, undergoing review and approval by the Institutional Review

119 Board of the HealthPartners Institute in Minneapolis, MN (registration A11-136). All the

120 participants completed an informed consent form before their enrollment.

\section{Measure: OIDP}

122 The OIDP is a widely used socio-dental indicator, that measures the impact of poor oral

123 health on individuals' performance of physical, psychological, and social performances.

124 Adulyanon et al. originally developed the OIDP in the adult general population in Thailand

125 [Adulyanon \& Sheiham, 1997]. Since then, psychometric soundness of the OIDP has been

126 supported in different countries [Åstrøm et al.,2003, 2005; Dorri et al., 2007; Erić et al., 2012;

127 Jung et al., 2008; Kida et al., 2006; Lajnert et al., 2016; Lawal et al., 2013; Montero et al., 2008;

128 Naito et al., 2007; Ostberg et al., 2008; Purohit et al., 2012]. There are 8 items or daily

129 performances on the OIDP. See Table 1 for the list of daily performances assessed in the OIDP.

130 There are 8 items or questions asked of respondents about whether their teeth, mouth or denture

131 caused them difficulty with any of the 8 daily performances within the last 6 months. Therefore,

132 OHRQoL is a negative concept, and the sum scores will be problem indices. If respondents

133 indicate "yes" to problems with teeth, mouth or denture having an impact on any of the daily

134 performances, then they answer questions about the frequency and severity of the impact of

135 dental problems on that performance. Table 2 shows specific frequency and severity questions

136 corresponding to each OIDP performance. Both frequency and severity scores are derived

137 separately. The last row in Table 2 shows overall scoring method for the OIDP and Table 3

138 shows criteria of frequency score.

*** Insert OIDP description Tables (Tables 1-3) here***

140 As shown in Table 2 under \#2 with specific criteria seen in Table 3, the OIDP provides

141 respondents with two ways to report frequency with which problems associated with mouth,

142 teeth, or denture impact on a particular performance. If the impact occurs regularly, respondents

143 report on the frequency over the past six months ranging from "Never" to "Every or nearly every

144 day". If the impact was restricted to a period of time (i.e., a spell), respondents report on how 
145 many days they suffered the impact in the past 6 months ranging from " 0 " to more than 3

146 months. As shown in Table 2 under \#3 the perceived severity of the impact of problems with

147 mouth, teeth, or dentures on any of the 8 performances was assessed by asking the respondents

148 to rate the severity of the impact on a scale from 0 to 5 , where $0=$ "no effect" and $5=$ "very

149 severe effect". Then, an individual score for the impact of problems with teeth, mouth or

150 dentures on each performance is calculated by multiplying the value for frequency with the

151 value for severity. The total score is the sum of all individual scores divided by the maximum

152 possible score (e.g., 8 performances $* 5$ frequency scores $* 5$ severity score $=200$ ) [Slade $\&$

153 Spencer, 1994; Adulyanon \& Sheiham, 1997]. See the last row of Table 2 for the OIDP scoring 154 method.

155 Correlation Analysis

156 We assessed correlations between the frequency and severity rating scales for: (a) all

157 OIDP items, (b) OIDP sum scores, and (c) severity and frequency scales as latent variables.

(1) Correlation analysis for OIDP items

We obtained polychoric correlation coefficients between each OIDP item's frequency and severity scales and their $95 \%$ confidence intervals. Polychoric correlation analysis is an appropriate correlation analysis method for ordinal data. We followed Cohen's (1988) criteria [Cohen, 1988] to interpret the magnitude of correlation coefficients $(r=0.10, r=0.30$, and $r=$ 0.50 represent small, moderate, and large magnitudes).

(2) Correlation analysis for the sum scores

We also summed up the individual item severity and frequency scores to derive two separate total scores for the OIDP frequency and severity scales. We then computed the correlation coefficient and its $95 \%$ confidence interval for these scores. In addition, we plotted the two total scores against each other to provide a visual representation of the strength of their relationship.

(3) Correlation analysis using latent variable modeling

We fit a structural equation model (SEM) to the OIDP data. In SEM, we relate frequency and severity items to latent variables, which corrects for attenuation due to measurement error

173 [Bedeian, Day, \& Kelloway, 1997]. We estimated the correlation between the two latent 
174 variables and determined if the correlation is close to one using a threshold of 0.85 [Clark \&

175 Watson, 1995; Kline, 2011].

176 Specifically, we fit a categorical SEM or an item factor analysis model wherein

177 diagonally weighted least squares (DWLS) estimation was applied when fitting the model to the

178 polychoric correlation matrix. Since SEM is a model-based approach, we evaluated model fit

179 using the chi-square test, root mean square error of approximation (RMSEA) and incremental fit

180 indices including comparative fit index (CFI) and Tucker-Lewis index (TLI). We assessed

181 model fit based on the commonly applied guidelines: RMSEA: $\leq 0.06$; and CFI, TLI: $\geq 0.95$ [John

182 et al., 2014; Hu \& Bentler, 1999].

\section{Results}

184 Participant characteristics

185 Our sample consisted a total of 873 study participants. There were more females $(n=545)$ than 186 males $(\mathrm{n}=328)$ participating in the studies. The mean age of the participants was $53.03 \pm 15.71$ (range 187 22-97) years.

188 Descriptive data for severity and frequency scores

A total of 835 study participants responded to all items in the severity scale (i.e.,

190 observations without missing values in the severity scale), with a mean score of 8.04 and

191 standard deviation of 8.12. While 773 participants responded to all items in the frequency scale

192 (i.e., observations without missing values in the frequency scale), with a mean score of 10.27 and

193 standard deviation of 9.11. A total of 762 participants responded to all items in both severity and

194 frequency scales and were thus considered in our analysis. We have summarized the prevalence

195 of the response categories in Table 4.

*** Insert Prevalence of Response Category Table (Table 4) here***

197 Correlation Analysis

(1) Correlations analysis for OIDP items

199

In Table 5 we present correlation coefficients and confidence intervals for the OIDP

200 items. Correlation coefficients for all items were above 0.50 , indicating a strong or large 
201 correlation between the frequency and severity scores. The coefficients were associated with

202 relatively narrow confidence intervals indicating precise estimates.

203

204

205

206

207

208

209

210

211

212

213

214

215

216

217

218

219

220

221

222

223

224

225

226

227

228

*** Insert Correlation Coefficients and Confidence Intervals Table (Table 5) here***

\section{(2) Correlation analysis for the sum scores}

The correlation estimate between the two summary scores was 0.85 (95\% CI: 0.82-0.86), indicating a strong correlation between the two rating scales. The estimates were supplemented by a visual representation of the strong correlation between the sum scores (as shown in Figure 1).

*** Insert Sum Score Plot (Figure 1) here***

(3) Correlation analysis using latent variable modeling

When we calculated the correlation coefficient in a latent variable model wherein the measurement error was considered, the value increased to 0.96 (95\% CI:0.93 - 1.00) (as shown in Figure 2). Measurement error accounts for the difference between the correlation of 0.85 and 0.96. However, we note that the model fit to the data was marginal $\left(\chi^{2}(103)=739.95, p<0.001\right.$, $\mathrm{RMSEA}=0.090, \mathrm{CFI}=0.944, \mathrm{TLI}=0.935)$. This is not surprising given that the same item stem is used in severity and frequency scales. This was also evidenced in the modification indices. Six out of 8 items (item 1, 2, 3, 4, 5, and 8) showed MI values greater than 10 .

$$
\text { *** Insert Latent Variable Modeling (Figure 2) here*** }
$$

\section{Discussion}

We found large correlations between severity and frequency scale scores for individual item scores and summary scores, presenting evidence of redundancy in the information provided by the two types of response formats. Dental researchers have previously used OIDP with only the frequency scale [Åstrøm et al., 2003]. Adulyanon and Sheiham investigated if multiplying the frequency score and severity score (that is, computing the traditional OIDP score) would add more information about impact than basing the scores on either frequency or severity responses alone [Adulyanon \& Sheiham,1997]. They applied three multiple regression models to predict DMFT (i.e., the sum of the number of permanent teeth that were decayed, missing due to caries, and filled) as well as the number of sextants with deep periodontal pockets. They observed that 
229 the improvement of OIDP compared to either frequency or severity score used alone was not

230 statistically significant in the prediction test, hence they recommended using one of them for

231 improving simplicity and efficiency. Frequency was suggested as a better representative single

232 choice due to its better reproducibility compared with severity. Other researchers have also used

233 only the OIDP frequency scores to assess OHRQoL in adolescent [Åstrøm et al., 2003;

234 Shekhawat et al., 2021] and adult general [Åstrøm et al., 2005] and dental patient populations

235 [Lawal et al., 2013; Lajnert et al., 2016; Ostberg et al., 2008; Gülcan et al., 2014] across the

236 world. Contrarily, in a recent study, Amilani et al. used only the OIDP severity scores, because

237 they found in a preliminary assessment that their participants' responses for the severity scale

238 were more consistent compared to their responses for the frequency scale [Amilani et al., 2020].

239 Previous researchers have also recommended use of frequency scales for other patient-

240 reported outcome measures. Chang et al. evaluated the comparability of the 5-point intensity and

241 frequency self-report rating scales for the questions from the Functional Assessment of Chronic

242 Illness Therapy (FACIT)-Fatigue 13-item scale [Chang et al., 2003]. Data from patients suffering

243 from cancer, stroke, and HIV were calibrated separately to fit an item response theory-based

244 rating scale model (RSM). The authors found no mean raw score difference between intensity

245 and frequency rating scales, and a high correlation $(r=.86, p<.001)$ between the intensity and

246 frequency scores indicating their essential equivalence. The authors concluded that it was

247 difficult to justify assessing both the response scales due to the essentially equivalent estimates

248 they derived. However, they preferred the frequency response scaling as it provided fuller

249 coverage of the fatigue continuum. Hatt et al. compared three different types of rating scales

250 when administering potential questionnaire items to children with eye disorders and their parents

251 [Hatt et al., 2017]. Children and parents in the study preferred the frequency scale over the

252 difficulty and severity scales. In another study, Krabbe \& Forkmann compared the usability of

253 frequency and intensity terms as verbal anchors of self-report scales in patients suffering from a

254 depressive episode [Krabbe \& Forkmann, 2012]. They found that frequency terms may have a

255 slight advantage over intensity terms with regard to intraindividual stability across time so it

256 might be advisable to use frequency terms when designing a self-report instrument that is

257 intended to be applied in longitudinal assessments [Krabbe \& Forkmann, 2012].

258 As mentioned before, there are advantages to using both frequency and severity scores; it

259 helps minimize subjective clinical judgment, creates a wider and finer grading scale for 
260 assessment of oral diseases impacts, and enables clinicians and researchers to assess the impact

261 of dental treatment on both symptom dimensions [Elhai et al., 2006]. However, our study

262 findings in conjunction with findings from previous studies mentioned above, suggest that both

263 the scales provide redundant information and using one scale only can be considered to improve

264 simplicity and efficiency. While the frequency response scale helps capture mild impacts that

265 occur more frequently, the severity response scale helps capture more severe impacts that occur

266 less frequently. Although more methodological research is needed to establish as to which

267 response scale is better, currently there is more evidence to support the use of the frequency scale 268 only in clinical and research settings.

\section{Strengths and Limitations}

270

271

272

273

274

275

276

277

278

279

280

281

282

283

284

285

286

287

288

289

The robustness of our study findings are supported by the use of a large sample of dental patients. We applied multiple analytical methodologies based on the classical methods and SEM at the scale and item levels to obtain a comprehensive view of the correlations between the frequency and severity response categories. Use of the SEM model allowed us to relate frequency and severity scales as latent variables while accounting for measurement error [Tarka, 2018]. We derived large correlations through the different analyses, which supports the preciseness of the study findings. Our study methodology can be applied to evaluating the response scales of other dPROMs which require patients to respond in terms of both frequency and severity.

In spite of the strengths, there were few limitations to our study. We acknowledge that the study findings are limited by the instrument (or dPROM) we chose to examine (OIDP). Although previous evidence suggests strong correlations among the OHRQoL instruments [Robinson et al., 2003; John et al., 2016], further research is needed to determine whether the findings from the current study would apply to other OHRQoL instruments. The correlation analyses in our study provide evidence of strong relationships between frequency and severity response scales, and the use of the frequency scale is recommended based on prior evidence [Adulyanon \& Sheiham, 1997; Åstrøm et al., 2003; Chang et al., 2003; Krabbe \& Forkmann, 2012]. Yet, the evidence regarding the preferred response scaling is mixed and more methodological work is needed to provide evidence that specifically supports the use of the frequency scale. For instance, Krabbe and Forkmann used a longitudinal study design, which 
290 supported the conclusion that frequency terms showed slightly greater stability across time.

291 Change scores for the two OIDP scales should be compared as well as comparing the strength of

292 the relationship of each to an external criterion.

\section{Significance of the study; Recommendations for research and practice}

294

295

296

297

298

299

300

301

302

303

304

305

306

307

308

309

310

311

312

313

314

315

316

317

318

319

The findings from our study support application of the OIDP frequency rating scale in clinical and research settings. The OIDP frequency scores had a strong correlation with the OIDP severity scores. Our findings align with previous research studies that have successfully adapted and applied the OIDP frequency scale to different cultural groups and contexts [Adulyanon \& Sheiham, 1997; Åstrøm et al., 2003, 2005; Lawal et al., 2013; Ostberg, Andersson, \& Hakeberg, 2008; Gülcan et al., 2014; Shekhawat et al.,2021]. The evidence from our study supporting the use of OIDP frequency rating scale is of great practical value for clinicians. dPROMs like OIDP, with more than one type of rating scales are more time-consuming and burdensome for patients, as they require patients to recall the frequency as well as the severity of the perceived impact of an oral disease [Reissmann, 2021]. Use of one response scale rather than two also will simplify the analysis and interpretation of the OIDP scores. Getting buy-in from dental providers and patients is necessary to capture dPROs more commonly and regularly [John et al., 2018; Paulson et al., 2021]. Less time-consuming dPROMs with one response scale would be more appealing to clinicians who typically have several demands on their time.

Currently, there is no consensus on the most appropriate rating scale for the OIDP and our study offers promising evidence for applying the frequency impact format in clinical practice and research. OIDP correlates well with other OHRQoL questionnaires, that is, GOHAI and OHIP as they all measure the same construct [Robinson et al., 2003; John et al., 2016; Oliveira et al., 2005]. OHIP and GOHAI, two of the most widely-used OHRQoL instruments, also use the frequency impact format, attesting to the research and clinical community's interest and support for the frequency impact. Use of a frequency scale also would help to standardize assessment of outcomes across dental and medical treatments as patient-reported outcome measures used in medicine often employ frequency response scales [Chang et al., 2003; Krabbe \& Forkmann, 2012].

Although further methodological work is needed, our study findings paves way for standardization of OHRQoL questionnaires and encourages the preferred use of frequency 
320 response scaling. Simple, short, and effective standardized questionnaires would make the

321 sharing and interpretation of results from the OIDP (and other dPROMs) within the dental

322 community more feasible [Palaiologou \& Kotsakis, 2020]. Regular assessment of dPROs would

323 improve dentist-patient communications [Palaiologou \& Kotsakis, 2020] and value-based oral

324 health care [Listl, 2019], eventually advancing evidence-based dental practice.

325 Conclusion

326 Our study findings and prior research support the use of the OIDP frequency scores only 327 in clinical and research settings, as they are highly correlated with the OIDP severity scores. Use 328 of only one response scale with OIDP would lower burden for patients and clinicians as well as 329 researchers. The direct evidence in this study from a head-to-head comparison of frequency and 330 severity oral impact format, indicated that severity is not superior compared to frequency, and 331 that one of the most widely OHRQoL instruments can be successfully applied with only one 332 response scale. The frequency impact format seems to be the preferred impact quality for OIDP, 333 specifically, and for dPROMs in general.

335 Declarations

336 Funding The National Institute of Dental and Craniofacial Research of the National Institutes of 337 Health, USA, under Award Numbers R01DE022331 and R01DE028059, supported the study.

338 Ethical approval and consent to participate

339 This research was conducted in accordance with accepted ethical standards for human-patient

340 research practice, undergoing review and approval by the Institutional Review Board of the

341 HealthPartners Institute in Minneapolis, MN (registration A11-136). All the participants

342 completed an informed consent form before their enrollment.

\section{Consent for publication}

344 Not applicable

345 Availability of data and materials

346 The datasets during and/or analysed during the current study available from the corresponding 347 author on reasonable request.

348 Competing interests 
349 The authors declare that there is no conflict of interest that could be perceived as prejudicing the

350 impartiality of the research reported.

351 Authors' contributions

352 SP, MJ, SC, and SK contributed to the study design, data analysis, interpretation, and drafting

353 the manuscript.

354 Acknowledgments

355 Not applicable

356

357

358

359

\section{References}

Adulyanon S, Sheiham A. 1997. Oral Impacts on Daily Performances. In: Slade GD, editor. Measuring oral health and quality of life. Chapel Hill: University of North Carolina.

Åstrøm AN, Okullo I. 2003. Validity and reliability of the Oral Impacts on Daily Performance

362 (OIDP) frequency scale: A cross-sectional study of adolescents in Uganda. BMC Oral Health 3(1):5 DOI: 10.1186/1472-6831-3-5.

Astrøm AN, Haugejorden O, Skaret E, Trovik TA, Klock KS. 2005. Oral Impacts on Daily Journal of Oral Sciences 113(4):289-296 DOI: 10.1111/j.1600-0722.2005.00225.x. structural equation models: Some important reminders. Educational and Psychological Measurement 57(5): 785- 799 DOI:10.1177/0013164497057005004 symptoms be scaled for intensity, frequency, or both? Palliative and Supportive Care 1(1):51-60 DOI:10.1017/S1478951503030049.

Cohen J. 1988. Statistical Power Analysis for the Behavioral Sciences. New York, NY:

375 Clark LA, Watson D. 1995. Constructing validity: Basic issues in objective scale development. Psychological Assessment 7(3):309-319. DOI:10.1037/1040-3590.7.3.309

Dorri M, Sheiham A, Tsakos G. 2007. Validation of a Persian version of the OIDP index. $B M C$ Oral Health 7:2 DOI: 10.1186/1472-6831-7-2. 
383 Erić J, Stančić I, Sojić LT, Jelenković Popovac A, Tsakos G. 2012. Validity, and reliability of 384 the Oral Impacts on Daily Performance (OIDP) scale in the elderly population of Bosnia and 385 Herzegovina. Gerodontology 29(2):e902-e908. DOI:10.1111/j.1741-2358.2011.00584.x.

386 Gülcan F, Nasir E, Ekbäck G, Ordell S, Åstrøm AN. 2014. Change in Oral Impacts on Daily 387 Performances (OIDP) with increasing age: Testing the evaluative properties of the OIDP 388 frequency inventory using prospective data from Norway and Sweden. BMC Oral Health 14:59 389 DOI: $10.1186 / 1472-6831-14-59$

390 Hatt SR, Leske DA, Wernimont SM, Birch EE, Holmes JM. 2017. Comparison of rating 391 scales in the development of patient-reported outcome measures for children with eye 392 disorders. Strabismus 25(1):33-38 DOI:10.1080/09273972.2016.1276941.

393 Hu L, Bentler PM. 1999. Cutoff criteria for fit indexes in covariance structure analysis:

394 Conventional criteria versus new alternatives. Structural Equation Modeling. 6:1-55.

395 John MT, Feuerstahler L, Waller N, Baba K, Larsson P, Celebić A, Kende D, Rener-Sitar

396 K, Reissmann DR. 2014. Confirmatory factor analysis of the Oral Health Impact

397 Profile. Journal of Oral Rehabilitation.41(9):644-652 DOI:10.1111/joor.12191.

398 John MT, Reissmann DR, Čelebić A, Baba K, Kende D, Larsson P, Rener-Sitar K. 2016.

399 Integration of oral health-related quality of life instruments. Journal of Dentistry. 53:38-43.

400 John MT. 2018. Health outcomes reported by dental patients. Journal of Evidence Based Dental 401 Practice.18(4):332-335 DOI:10.1016/j.jebdp.2018.09.001.

402 Jung SH, Ryu JI, Tsakos G, Sheiham A. 2008. A Korean version of the Oral Impacts on Daily 403 Performances (OIDP) scale in elderly populations: Validity, reliability, and prevalence. Health 404 and Quality of Life Outcomes 6:17 DOI:10.1186/1477-7525-6-17.

405 Kida IA, Astrøm AN, Strand GV, Masalu JR, Tsakos G. 2006. Psychometric properties and 406 the prevalence, intensity and causes of oral impacts on daily performance (OIDP) in a population 407 of older Tanzanians. Health and Quality of Life Outcomes 4:56 DOI:10.1186/1477-7525-4-56.

408 Kline RB. 2011. Principles and practice of structural equation modeling (3rd ed.). Guilford 409 Press.

410 Krabbe J, Forkmann T. 2012. Frequency vs. intensity: Which should be used as anchors for 411 self-report instruments? Health and Quality of Life Outcomes. 10:107. DOI: 10.1186/1477412 7525-10-107.

413 Lajnert V, Gržić R, Radica N, Šnjarić D, Špalj S. 2016. Translation and validation of the 414 Croatian version of the Oral Impacts on Daily Performances (OIDP) scale. Vojnosanitetski 415 pregled 73(9):811-816 DOI:10.2298/VSP140829136L.

416 Larsson P, John MT, Nilner K, Bondemark L, List T. 2010. Development of an Orofacial 417 Esthetic Scale in prosthodontic patients. International Journal of Prosthodontics. 23(3):249-256. 
418 Lawal FB, Taiwo JO, Arowojolu MO. 2013. Validation of the oral impact on daily

419 performance frequency scale in Ibadan, Nigeria. African Journal of Medicine and Medical

420 Sciences 42(3):215-22 PMID: 24579382.

421 Listl S. 2019. Value-based oral health care: Moving forward with dental patient-reported

422 outcomes. Journal of Evidence Based Dental Practice. 19(3):255-259

423 DOI:10.1016/j.jebdp.2019.101344.

424 Mittal H, John MT, Sekulić S, Theis-Mahon N, Rener-Sitar K. 2019. Patient-reported 425 outcome measures for adult dental patients: A systematic review. Journal of Evidence Based 426 Dental Practice.19(1):53-70 DOI:10.1016/j.jebdp.2018.10.005.

427 Montero J, Bravo M, Albaladejo A. 2008. Validation of two complementary oral-health related 428 quality of life indicators (OIDP and OSS 0-10) in two qualitatively distinct samples of the 429 Spanish population. Health and Quality of Life Outcomes 6:101 DOI: 10.1186/1477-7525-6-101.

430 Naito M, Suzukamo Y, Ito HO, Nakayama T. 2007. Development of a Japanese version of the 431 Oral Impacts on Daily Performance (OIDP) scale: A pilot study. Journal of Oral Sciences 432 49(4):259-264. DOI:10.2334/josnusd.49.259.

433 Oliveira BH, Nadanovsky P. 2005. Psychometric properties of the Brazilian version of the Oral 434 Health Impact Profile-short form. Community Dentistry and Oral Epidemiology 33(4):307-14.

435 DOI: $10.1111 / \mathrm{j} .1600-0528.2005 .00225 . \mathrm{x}$

436 Organisation for Economic Co-operation and Development (OECD). 2013. OECD

437 Guidelines on measuring subjective well-being. Methodological considerations in the

438 measurement of subjective well-being. Paris.

439 Ostberg AL, Andersson P, Hakeberg M. 2008. Cross-cultural adaptation, and validation of the 440 oral impacts on daily performances (OIDP) in Swedish. Swedish Dental Journal 32(4):187-195.

441 Paulson DR, Pattanaik S, Chanthavisouk P, John MT. 2021. Including the patient's oral 442 health perspective in evidence-based decision-making. Bundesgesundheitsbl 64: 959-966 443 DOI:10.1007/s00103-021-03375-0.

444 Palaiologou A, Kotsakis GA. 2020. Dentist-Patient communication of treatment outcomes in 445 periodontal practice: A need for dental patient-reported outcomes. Journal of Evidence Based 446 Dental Practice 20(2):101443. DOI:10.1016/j.jebdp.2020.101443

447 Purohit BM, Singh A, Acharya S, Bhat M, Priya H. 2012. Assessment and validation of the 448 oral impact on daily performance (OIDP) instrument among adults in Karnataka, South 449 India. Community Dental Health. 29(3):203-208.

450 Reissmann DR. 2021. Methodological considerations when measuring oral health-related 451 quality of life. Journal of Oral Rehabilitation.48:233-245 DOI:10.1111/joor.12983

452 Robinson PG, Gibson B, Khan FA, Birnbaum W. 2003. Validity of two oral health-related quality 453 of life measures. Community Dentistry and Oral Epidemiology.31(2):90-9. 
454 Shekhawat KS, Samuel SR, Chauhan A. 2021. Frequency of Oral Impacts on Daily 455 Performances, and dental pain among indigenous adolescents of Himalayas (Leh, Ladakh): A 456 Cross-Sectional Study. Oral Health \& Preventive Dentistry.19(1):115-120 DOI:

457 10.3290/j.ohpd.b965717.

458 Simancas-Pallares M, John MT, Prodduturu S, Rush WA, Enstad CJ, Lenton P. 2018.

459 Development, validity, and reliability of the Orofacial Esthetic Scale - Spanish version. Journal 460 of Prosthodontic Research.62(4):456-461 DOI:10.1016/j.jpor.2018.05.003.

461 Simancas-Pallares M, John MT, Enstad C, Lenton P. 2020. The Spanish Language 5-Item

462 Oral Health Impact Profile. International Dental Journal.70(2):127-135

463 DOI:10.1111/idj.12534

464 Slade GD, Spencer AJ. 1994. Development and evaluation of the Oral Health Impact

465 Profile. Community Dental Health.11(1):3-11.

466 Tarka P. 2018. An overview of structural equation modeling: Its beginnings, historical

467 development, usefulness, and controversies in the social sciences. Quality and Quantity 52:313-

468354 DOI: 10.1007/s11135-017-0469-8.

469

470

471

472

473

474

475

476

477

478

479 


\section{Table 1 (on next page)}

Performances assessed in the Oral Impacts on Daily Performances 
1 Table 1. Performances assessed in the Oral Impacts on Daily Performances

\section{List of Daily Performances}

Physical Performances
a. Eating and enjoying food
b. Speaking and pronouncing clearly
c. Cleaning teeth

Psychological Performances

d. Sleeping and relaxing

e. Smiling, laughing, and showing teeth without embarrassment

f. Maintain usual emotional state without being irritable

\section{Social Performances}

g. Carrying out major work or social role

h. Enjoying contact with people

2

3

4

5

6

7

8

9

10 


\section{Table 2 (on next page)}

Severity- and frequency- related questions and the scoring method in the Oral Impacts on Daily Performances 
1 Table 2. Severity- and frequency- related questions and the scoring method in the Oral

2 Impacts on Daily Performances

OIDP questions asked for each daily performance or item

1) In the past 6 months, have problems with mouth, teeth, or dentures caused you any difficulty in performance (see Table 1 for the list of performances)? If yes,

2a) Have you had this difficulty in (performance) on a regular/periodic basis or for a period/spell?

-If ability restricted on a "regular/periodic basis",

$2 \mathrm{~b}$ ). During the past six months how often have you had this difficulty?*

-or, if ability restricted "on a period/spell"

$2 \mathrm{c})$. For how much of the past 6 months have you had this difficulty?*

3) And using a scale from 0 to 5 , where 0 is no effect and 5 is a very severe effect, which number would you say reflects what the difficulty in (performance) had on your daily life?

Scoring method for Oral Impact on Daily Performances

OIDP score $=$

[(frequency score* of "Eating" $\times$ severity score* of "Eating") +

(frequency of "Speaking" $\times$ severity of "Speaking") +

(frequency of "Cleaning teeth" $\times$ severity of "Cleaning teeth") +

(frequency of "Sleeping" $\times$ severity of "Sleeping") +

(frequency of "Smiling" $\times$ severity of "Smiling") +

(frequency of "Emotional stability" × severity of "Emotional stability") +

(frequency of "Major role" × severity of "Major role") +

(frequency of "Contact with people" $\times$ severity of "Contact with people") ]

$\times 100 / 200 \dagger$

$4 * *$ Scores range from 0 to 5

5 *Please see Table 2 for Responses and scores for answers to $2 \mathrm{~b}$ and $2 \mathrm{c}$

$6 \uparrow$ maximum possible score [Sum of 8 performances score $\times 5$ frequency score $\times$

75 severity score $]=200$

8 


\section{Table 3 (on next page)}

Criteria of frequency score of affected performances over past six months 
1 Table 3. Criteria of frequency score of affected performances over past six months

\begin{tabular}{lll}
\hline $\begin{array}{l}\text { Frequency (for people } \\
\text { affected on a regular or } \\
\text { periodic basis) }\end{array}$ & $\begin{array}{l}\text { Duration (for people affected } \\
\text { for a period/spell) }\end{array}$ & Score \\
\hline $\begin{array}{ll}\text { Never affected in past } 6 \text { months } \\
\text { Less than once a month }\end{array}$ & 0 days & 0 \\
Once or twice a month & Up to 5 days in total & 1 \\
Once or twice a week & Up to 15 days in total & 2 \\
3-4 times a week & Up to 30 days in total & 3 \\
Every or nearly every day & Up to 3 months in total & 4 \\
\hline
\end{tabular}

2 
Table 4 (on next page)

Prevalence of response category [\%] 
1 Table 4. Prevalence of response category [\%]

\begin{tabular}{|c|c|c|c|c|c|c|c|c|c|c|c|c|c|c|c|c|c|}
\hline \multirow[b]{2}{*}{$\begin{array}{c}\text { Response } \\
\text { Options }\end{array}$} & \multicolumn{8}{|c|}{ Severity item } & \multicolumn{9}{|c|}{ Frequency item } \\
\hline & 1 & 2 & 3 & 4 & 5 & 6 & 7 & 8 & & 1 & 2 & 3 & 4 & 5 & 6 & 7 & 8 \\
\hline 0 & 31 & 64 & 40 & 56 & 39 & 63 & 72 & 65 & $\mathbf{0}$ & 22 & 58 & 34 & 49 & 33 & 56 & 65 & 58 \\
\hline 1 & 25 & 15 & 24 & 18 & 19 & 15 & 11 & 12 & 1 & 19 & 10 & 19 & 15 & 14 & 13 & 11 & 11 \\
\hline 2 & 13 & 6 & 12 & 9 & 11 & 7 & 5 & 6 & 2 & 15 & 7 & 13 & 11 & 11 & 7 & 5 & 7 \\
\hline 3 & 15 & 6 & 12 & 7 & 11 & 6 & 4 & 6 & 3 & 12 & 5 & 9 & 6 & 9 & 6 & 3 & 5 \\
\hline 4 & 7 & 4 & 5 & 4 & 7 & 3 & 3 & 4 & 4 & 10 & 5 & 7 & 6 & 6 & 4 & 3 & 4 \\
\hline 5 & 6 & 2 & 5 & 2 & 11 & 2 & 2 & 4 & 5 & 14 & 7 & 13 & 5 & 19 & 4 & 3 & 6 \\
\hline Miss & 2 & 3 & 2 & 3 & 2 & 4 & 4 & 4 & Miss & 6 & 9 & 6 & 8 & 7 & 10 & 10 & 9 \\
\hline
\end{tabular}


Table 5 (on next page)

Correlation coefficients and confidence intervals for OIDP items 
1 Table 5. Correlation coefficients and confidence intervals for OIDP items

2

3

\section{OIDP Items}

Correlation (95\% confidence interval)

Item 1: Eating and enjoying food

$0.75(0.72-0.78)$

Item 2: Speaking and pronouncing clearly

$0.82(0.80-0.84)$

Item 3: Cleaning teeth

$0.74(0.71-0.78)$

Item 4: Sleeping and relaxing

$0.82(0.79-0.84)$

Item 5: Smiling, laughing, and showing teeth without

$0.83(0.81-0.85)$

embarrassment

Item 6: Maintain usual emotional state without being irritable

$0.74(0.70-0.77)$

Item 7: Carrying out major work or social role

$0.78(0.75-0.81)$

Item 8: Enjoying contact with people

$0.80(0.77-0.82)$

Summary score

$0.85(0.82-0.86)$ 
Table 6(on next page)

Plot representing the correlation between the sum scores from the severity and frequency rating scales. 
1

2

Figure 1: Plot representing the correlation between the sum scores from the severity and frequency rating scales.

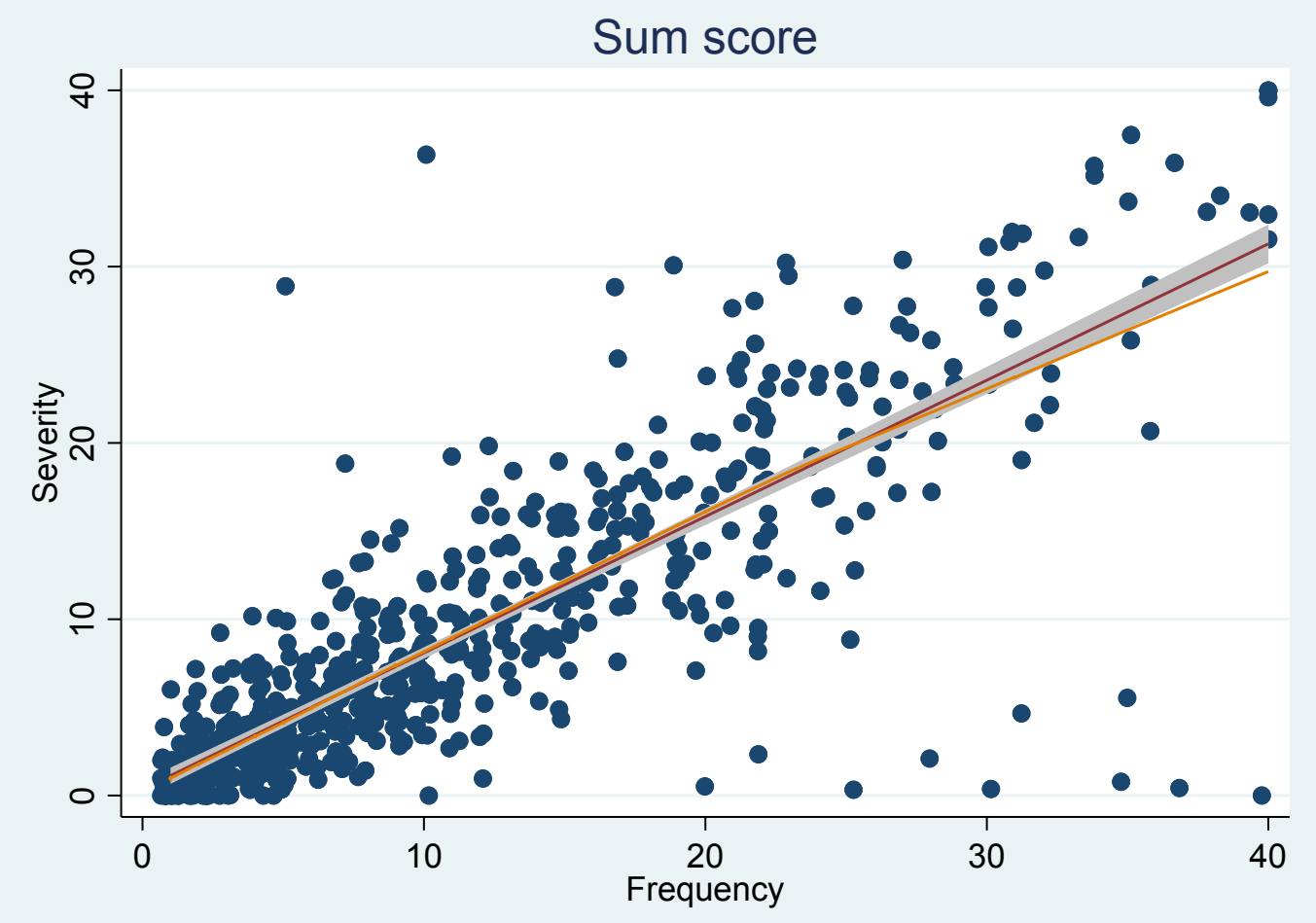


Table 7 (on next page)

Latent variable modeling between the two rating scales. 
Figure 2. Latent variable modeling between the two rating scales.

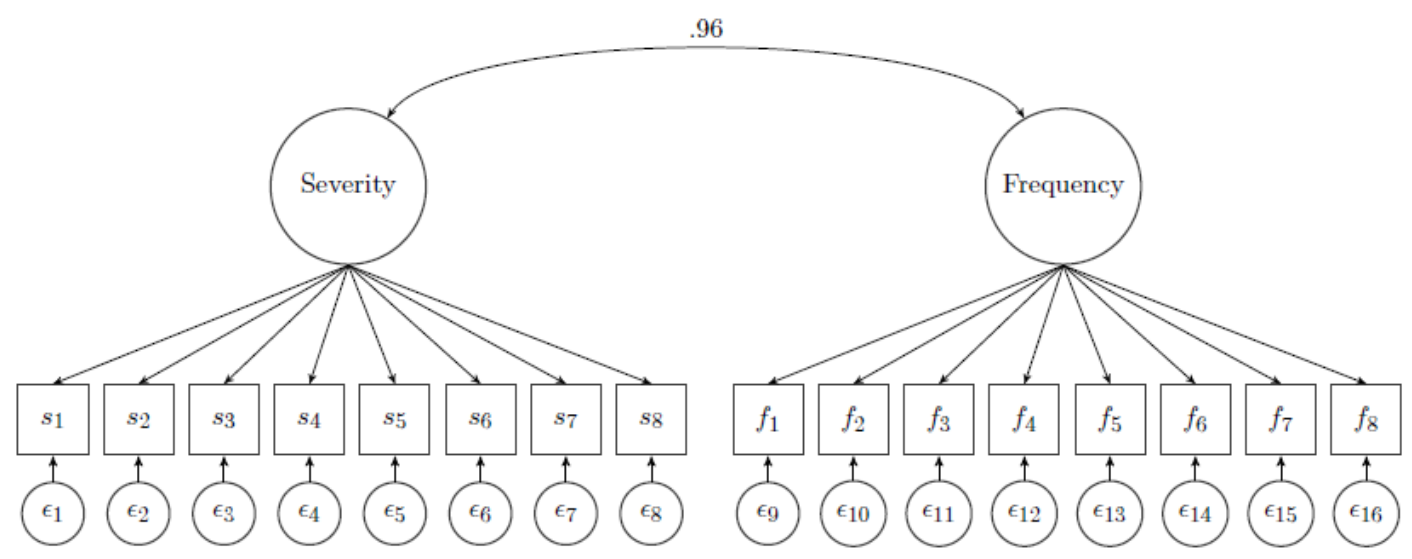

2 